\title{
REDUCTIVE COMPACTIFICATIONS OF SEMITOPOLOGICAL SEMIGROUPS
}

\author{
ABDOLMAJID FATTAHI, MOHAMAD ALI POURABDOLLAH, \\ and ABBAS SAHLEH
}

Received 10 February 2002

\begin{abstract}
We consider the enveloping semigroup of a flow generated by the action of a semitopological semigroup on any of its semigroup compactifications and explore the possibility of its being one of the known semigroup compactifications again. In this way, we introduce the notion of $E$-algebra, and show that this notion is closely related to the reductivity of the semigroup compactification involved. Moreover, the structure of the universal $E \mathscr{F}$-compactification is also given.
\end{abstract}

2000 Mathematics Subject Classification: 22A20, 43A60.

1. Introduction. A semigroup $S$ is called right reductive if $a=b$ for each $a, b \in S$, since $a t=b t$ for every $t \in S$. For example, all right cancellative semigroups and semigroups with a right identity are right reductive.

From now on, $S$ will be a semitopological semigroup, unless otherwise is stipulated. By a semigroup compactification of $S$ we mean a pair $(\psi, X)$, where $X$ is a compact Hausdorff right topological semigroup, and $\psi: S \rightarrow X$ is a continuous homomorphism with dense image such that, for each $s \in S$, the mapping $x \rightarrow \psi(s) x: X \rightarrow X$ is continuous. The $C^{*}$-algebra of all bounded complex-valued continuous functions on $S$ will be denoted by $\mathscr{C}(S)$. For $\mathscr{C}(S)$, the left and right translations, $L_{s}$ and $R_{t}$, are defined for each $s, t \in S$ by $\left(L_{s} f\right)(t)=f(s t)=$ $\left(R_{t} f\right)(s), f \in \mathscr{C}(S)$. The subset $\mathscr{F}$ of $\mathscr{C}(S)$ is said to be left translation invariant if for all $s \in S, L_{s} \mathscr{F} \subseteq \mathscr{F}$. A left translation invariant unital $C^{*}$-subalgebra $\mathscr{F}_{F}$ of $\mathscr{C}(S)$ is called $m$-admissible if the function $s \rightarrow T_{\mu} f(s)=\mu\left(L_{s} f\right)$ is in $\mathscr{F}_{F}$ for all $f \in \mathscr{F}$ and $\mu \in S^{\mathscr{F}}$ (where $S^{\mathscr{F}}$ is the spectrum of $\mathscr{F}$ ). Then the product of $\mu, v \in S^{\mathscr{F}}$ can be defined by $\mu \nu=\mu \circ T_{v}$ and the Gelfand topology on $S^{\mathscr{F}}$ makes $\left(\epsilon, S^{\mathscr{F}}\right)$ a semigroup compactification (called the $\mathscr{F}$-compactification) of $S$, where $\epsilon: S \rightarrow S^{\mathscr{F}}$ is the evaluation mapping.

Some $m$-admissible subalgebras of $\mathscr{b}(S)$, that we will need, are left multiplicatively continuous functions $\mathscr{L} \mathcal{M} \mathscr{C}$, distal functions $\mathscr{D}$, minimal distal functions $\mathcal{M D}$, and strongly distal functions $\mathscr{\mathscr { D }}$. We also write $\mathscr{G} \mathscr{P}$ for $\mathcal{M D} \cap \mathscr{G}$; and we define $\mathscr{L} \mathscr{\mathscr { L }}:=\{f \in \mathscr{C}(S) ; f(s t)=f(s)$ for all $s, t \in S\}$. For a discussion of the universal property of the corresponding compactifications of these function algebras see [1, 2]. 
2. Reductive compactifications and $E$-algebras. Let $(\psi, X)$ be a compactification of $S$, then the mapping $\sigma: S \times X \rightarrow X$, defined by $\sigma(s, x)=\psi(s) x$, is separately continuous and so $(S, X, \sigma)$ is a flow. If $\Sigma_{X}$ denotes the enveloping semigroup of the flow $(S, X, \sigma)$ (i.e., the pointwise closure of semigroup $\{\sigma(s, \cdot): s \in S\}$ in $\left.X^{X}\right)$ and the mapping $\sigma_{X}: S \rightarrow \Sigma_{X}$ is defined by $\sigma_{X}(s)=\sigma(s, \cdot)$ for all $s \in S$, then $\left(\sigma_{X}, \Sigma_{X}\right)$ is a compactification of $S$ (see [1, Proposition 1.6.5]).

One can easily verify that $\Sigma_{X}=\left\{\lambda_{x}: x \in X\right\}$, where $\lambda_{x}(y)=x y$ for each $y \in X$. If we define the mapping $\theta: X \rightarrow \Sigma_{X}$ by $\theta(x)=\lambda_{x}$, then $\theta$ is a continuous homomorphism with the property that $\theta \circ \psi=\sigma_{X}$. So $\left(\sigma_{X}, \Sigma_{X}\right)$ is a factor of $(\psi, X)$, that is $(\psi, X) \geq\left(\sigma_{X}, \Sigma_{X}\right)$. By definition, $\theta$ is one-to-one if and only if $X$ is right reductive. So we get the next proposition, which is an extension of the Lawson's result [3, Lemma 2.4(ii)].

Proposition 2.1. Let $(\psi, X)$ be a compactification of $S$. Then $\left(\sigma_{X}, \Sigma_{X}\right) \cong$ $(\psi, X)$ if and only if $X$ is right reductive.

A compactification $(\psi, X)$ is called reductive if $X$ is right reductive. For example, the $M \mathscr{D}$-, $\mathscr{G} \mathscr{P}$-, and $\mathscr{L} \mathscr{L}$-compactifications are reductive.

An $m$-admissible subalgebra $\mathscr{F}$ of $\mathscr{C}(S)$ is called an E-algebra if there is a compactification $(\psi, X)$ such that $\left(\sigma_{X}, \Sigma_{X}\right) \cong\left(\epsilon, S^{\mathscr{F}}\right)$. In this setting $(\psi, X)$ is called an EF्F-compactification of $S$. Trivially for every reductive compactification $(\psi, X), \psi^{*}(\mathscr{C}(X))$ is an $E$-algebra. But the converse is not, in general, true. For instance, for any compactification $(\psi, X), \sigma_{X}^{*}\left(\mathscr{C}\left(\Sigma_{X}\right)\right)$ is an $E$-algebra; however, it is possible that $\Sigma_{X}$ would be nonreductive, as the next example shows.

EXAMPLE 2.2. Let $S=\{a, b, c, d\}$ be the semigroup with the following multiplication table:

\begin{tabular}{c|cccc} 
& $a$ & $b$ & $c$ & $d$ \\
\hline$a$ & $a$ & $a$ & $a$ & $a$ \\
$b$ & $a$ & $a$ & $a$ & $c$ \\
$c$ & $a$ & $a$ & $a$ & $a$ \\
$d$ & $a$ & $c$ & $a$ & $b$
\end{tabular}

Then for the identity compactification $(i, X)$ of $S, \Sigma_{X}$ is not right reductive; in fact, $\lambda_{a} \neq \lambda_{b}$, however, $\lambda_{a t}=\lambda_{b t}$ for every $t \in S$.

LEMMA 2.3. If $(\psi, X)$ is a compactification satisfying $X^{2}=X$, then the compactification $\left(\sigma_{X}, \Sigma_{X}\right)$ is reductive.

Proof. Since $X^{2}=X$, for each $x_{1}, x_{2} \in X$, from $\lambda_{x_{1}} \lambda_{y}=\lambda_{x_{2}} \lambda_{y}$ for every $\lambda_{y} \in \Sigma_{X}$, it follows that $\lambda_{x_{1}}=\lambda_{x_{2}}$. So $\Sigma_{X}$ is right reductive.

Corollary 2.4. Let $s S$ (or $S s$ ) be dense in $S$, for some $s \in S$, then for every compactification $(\psi, X)$ of $S$, it follows that $X^{2}=X$ and so $\left(\sigma_{X}, \Sigma_{X}\right)$ is reductive. 
Now, we are going to construct the universal EF्F-compactification of $S$. For this end we need the following lemma.

LEMMA 2.5. Let $\mathscr{F}$ be an m-admissible subalgebra of $\mathscr{C}(S)$. Then $T_{v} f \in$ $\sigma_{S^{\mathscr{F}}}^{*}\left(\mathscr{C}\left(\Sigma_{S^{\mathscr{F}}}\right)\right)$ for all $f \in \mathscr{F}^{\mathscr{F}}$ and $v \in S^{\mathscr{L} M \mathscr{C}}$.

Proof. Since $\Sigma_{S^{\mathscr{F}}}=\left\{\lambda_{\mu}: \mu \in S^{\mathscr{F}}\right\}$, we can define $g: \Sigma_{S^{\mathscr{F}}} \rightarrow \mathbb{C}$ by $g\left(\lambda_{\mu}\right)=$ $\mu\left(T_{\nu} f\right)$, where $\mathbb{C}$ denotes the complex numbers. Since the mapping $\lambda_{\mu} \rightarrow \mu \nu$ : $\Sigma_{S^{\mathscr{F}}} \rightarrow S^{\mathscr{F}}$ is $p$-weak* continuous, $g$ is a bounded continuous function and it is easy to see that $\sigma_{S^{\mathscr{F}}}^{*}(g)=T_{v}(f)$. Therefore, $T_{v} f \in \sigma_{S^{\mathscr{F}}}^{*}\left(\mathscr{C}\left(\Sigma_{S^{\mathscr{F}}}\right)\right)$ for all $v \in S^{\mathscr{F}}$. If $\tilde{v} \in S^{\mathscr{L} \mathcal{M}}$ and $v$ is the restriction of $\tilde{v}$ to $\mathscr{F}$, then $T_{\tilde{v}} f=T_{v} f$ for all $f \in \mathscr{F}$. So the conclusion follows.

Proposition 2.6. Let $\mathscr{F}$ be an E-algebra. Then

$$
G_{\mathscr{F}}:=\left\{f \in \mathscr{L} \mathcal{M} \mathscr{C}: T_{v} f \in \mathscr{F} \forall v \in S^{\mathscr{L} M \mathscr{C}}\right\}
$$

is an $m$-admissible subalgebra of $\mathscr{C}(S)$ and $\left(\epsilon, S^{G \mathscr{F}}\right)$ is the universal EF्F-compactification of $S$.

Proof. It is easy to verify that $G_{\mathscr{F}}$ is an $m$-admissible subalgebra of $\mathscr{C}(S)$

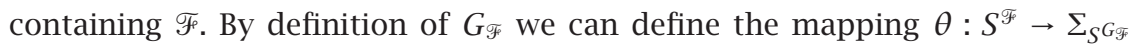
by $\theta(\mu)=\lambda_{\tilde{\mu}}$, where $\tilde{\mu}$ is an extension of $\mu$ to $S^{G_{\tilde{F}}}$. Clearly, $\theta$ is continuous and $\theta \circ \epsilon=\sigma_{S^{G \mathscr{F}}}$. Thus $\left(\epsilon, S^{\mathscr{F}}\right) \geq\left(\sigma_{S_{\mathscr{F}}}, \Sigma_{S^{G \mathscr{F}}}\right)$. On the other hand, since $\mathscr{F}_{\mathscr{F}}$ is an $E$-algebra, there exists a compactification $(\phi, Y)$ of $S$ such that $\left(\sigma_{Y}, \Sigma_{Y}\right) \cong$ $\left(\epsilon, S^{\mathscr{F}}\right)$ and $\mathscr{F}_{F}=\sigma_{Y}^{*}\left(\mathscr{C}\left(\Sigma_{Y}\right)\right)$. By Lemma 2.5, we have $T_{v} f \in \sigma_{Y}^{*}\left(\mathscr{C}\left(\Sigma_{Y}\right)\right)$, for each $v \in S^{\mathscr{L} \mathcal{M}}$ and each $f \in \phi^{*}(\mathscr{C}(Y))$. This means that $\phi^{*}(\mathscr{C}(Y)) \subset G_{\mathscr{F}}$ and so, by [1, Propositiion 1.6.7], $\left(\sigma_{Y}, \Sigma_{Y}\right) \leq\left(\sigma_{S} G_{\mathscr{F}}, \Sigma_{S} G_{\mathscr{F F}}\right)$. Therefore, $\left(\epsilon, S^{\mathscr{F}}\right) \cong$ $\left(\sigma_{S^{G \mathscr{F}}}, \Sigma_{S_{\mathscr{F}}}\right)$ and $\left(\epsilon, S^{G \mathscr{F}}\right)$ is an EF्F-compactification of $S$. Finally, if $(\psi, X)$ is

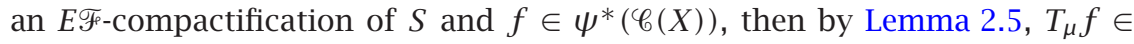
$\sigma_{X}^{*}\left(\mathscr{C}\left(\Sigma_{X}\right)\right)=\mathscr{F}$ for all $\mu \in S^{\mathscr{L M} \mathscr{C}}$. So $\psi^{*}(\mathscr{C}(X)) \subset G_{\mathscr{F}}$ and $(\psi, X) \leq\left(\epsilon, S^{G \mathscr{F}}\right)$.

EXAMPLES 2.7. (a) We have $G_{\mathcal{M D}}=\mathscr{D}$. To see this, if $f \in G_{\mathcal{M D}}$, then for all $\mu, \nu, \eta \in S^{\mathscr{M} \mathcal{C}}$ with $\eta^{2}=\eta$, we have $\mu \eta \nu(f)=\mu \eta\left(T_{v} f\right)=\mu\left(T_{v} f\right)=\mu \nu(f)$. So $f \in \mathscr{D}$. Also if $f \in \mathscr{D}$, then for all $\mu, \nu, \eta \in S^{\mathscr{M} \mathcal{M C}}$ with $\eta^{2}=\eta$, we have $\mu \eta\left(T_{v} f\right)=$ $\mu \eta \nu(f)=\mu \nu(f)=\mu\left(T_{v} f\right)$. That is, $T_{v} f \in M \mathscr{D}$ for all $v \in S^{\mathscr{L} M \mathscr{C}}$ and so $f \in G_{M \mathscr{D}}$ (see also [4, Lemma 2.2]).

(b) By a similar proof, we can show that $G_{\mathscr{g} \mathscr{P}}=\mathscr{\mathscr { D }}$ (see [4, Lemma 2.2 and Theorem 2.6]).

(c) Let $\mathscr{R}:=\{f \in \mathscr{L} \mathcal{M} \mathscr{C}(S): f(r s t)=f(r t)$ for $r, s, t \in S\}$. Clearly, $\mathscr{R}$ is an $m$-admissible subalgebra of $\mathscr{C}(S)$. If $f \in \mathscr{R}$ and $v \in S^{\mathscr{L} \mathcal{M} \mathscr{C}}$, then for each $r, s, t \in S$ we have $L_{r t} f(s)=f(r t s)=f(r s)=L_{r} f(s)$. So $T_{v} f(r t)=$ $v\left(L_{r t} f\right)=v\left(L_{r} f\right)=T_{v} f(r)$. That is, $T_{v} f \in \mathscr{L} \mathscr{L}$. On the other hand, if $f \in G_{\mathscr{L} \mathscr{I}}$, then $f(r s t)=\left(T_{\epsilon(t)} f\right)(r s)=\left(T_{\epsilon(t)} f\right)(r)=f(r t)$ and so $f \in \mathscr{R}$. Therefore, $G_{\mathscr{L} \mathscr{L}}=\mathscr{R}$. 
ACKNowledgment. In the end, we would like to thank Dr H. R. EbrahimiVishki for his valuable comments.

\section{REFERENCES}

[1] J. F. Berglund, H. D. Junghenn, and P. Milnes, Analysis on Semigroups, Function Spaces, Compactifications, Representations, Canadian Mathematical Society Series of Monographs and Advanced Texts, John Wiley \& Sons, New York, 1989.

[2] H. D. Junghenn, Distal compactifications of semigroups, Trans. Amer. Math. Soc. 274 (1982), no. 1, 379-397.

[3] J. D. Lawson, Flows and compactifications, J. London Math. Soc. (2) 46 (1992), no. 2, 349-363.

[4] M. A. Pourabdollah and A. Sahleh, Flows and universal compactifications, J. Sci. Islam. Repub. Iran 8 (1997), no. 4, 269-273.

Abdolmajid Fattahi: Department of Mathematics, Faculty of Science, Razi University, Kermanshah, Iran

E-mail address: abfattahi@yahoo.ca

Mohamad Ali Pourabdollah: Faculty of Mathematics, Ferdowsi University of Mashhad, P.O. Box 91775-1159, Mashhad, Iran

E-mail address: pourabd@math . um . ac. ir

Abbas Sahleh: Department of Mathematics, Guilan University, Rasht, Iran

E-mail address: sah7ehj@kd.gu .ac.ir 


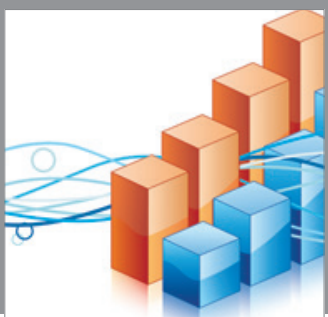

Advances in

Operations Research

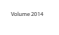

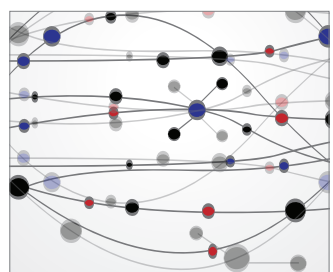

\section{The Scientific} World Journal
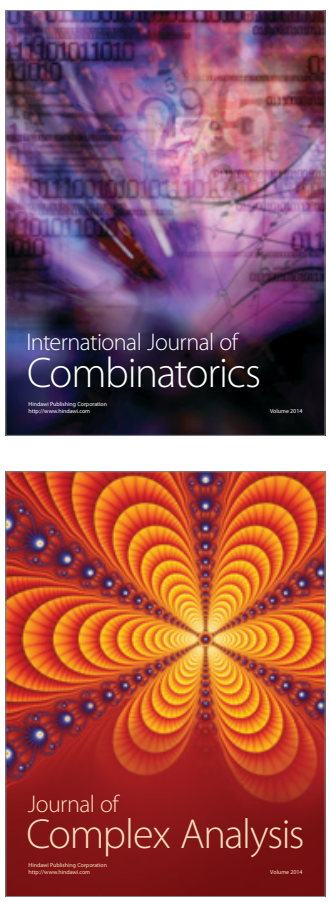

International Journal of

Mathematics and

Mathematical

Sciences
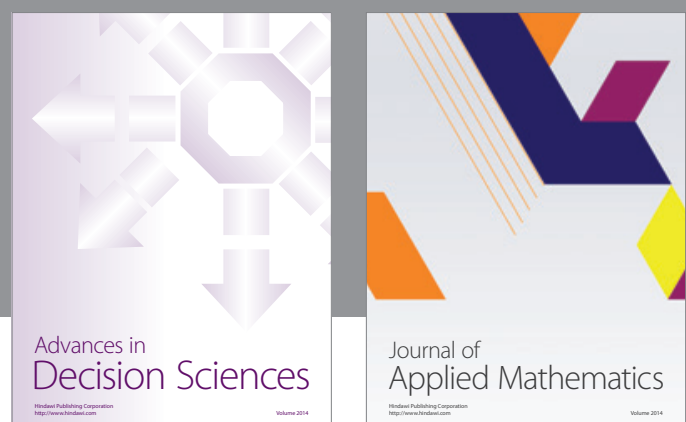

Journal of

Applied Mathematics
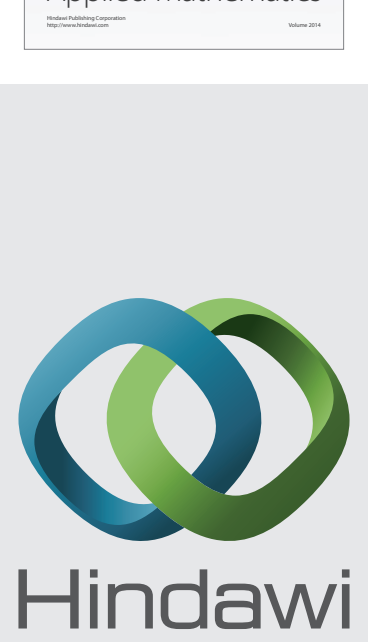

Submit your manuscripts at http://www.hindawi.com
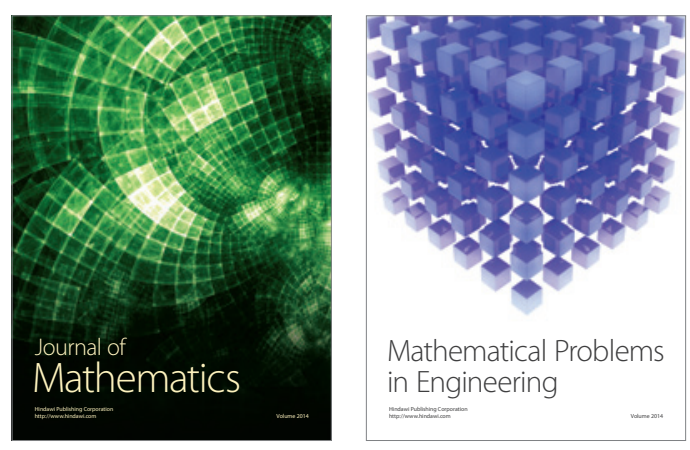

Mathematical Problems in Engineering
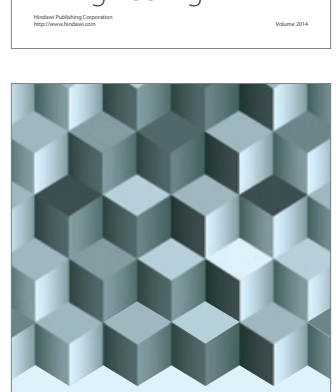

Journal of

Function Spaces
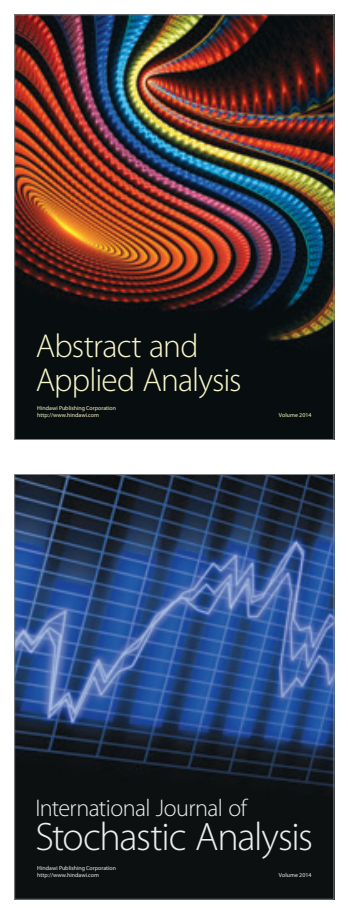

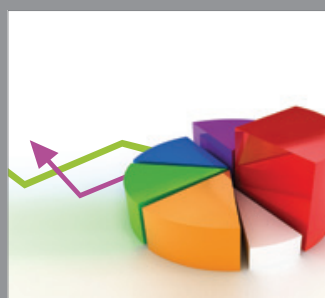

ournal of

Probability and Statistics

Promensencen
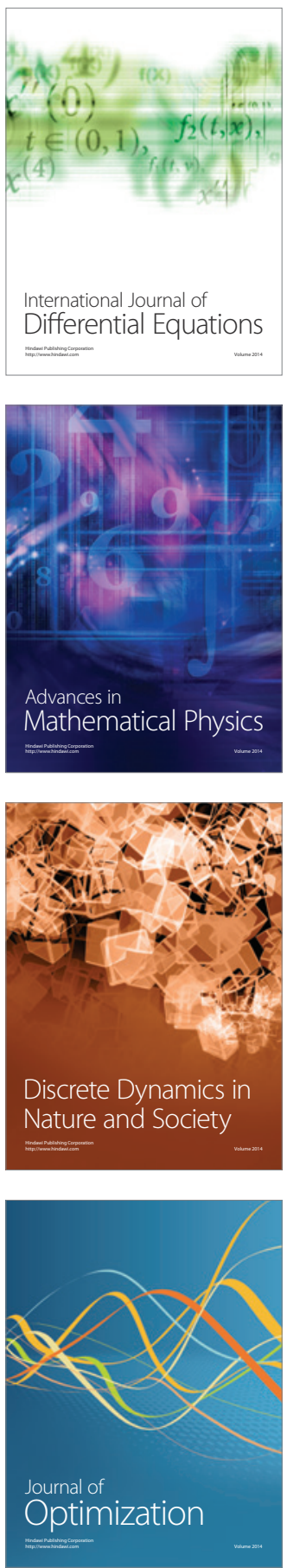